\title{
VIOLENCIA FAMILIAR CONTRA LAS PERSONAS ANCIANAS QUE SUFREN DEPENDENCIA Y ENFERMEDAD
}

\author{
MARÍA TERESA BAZO
}

Catedrática de Sociología. Universidad del País Vasco/EHU.

\section{RESUMEN}

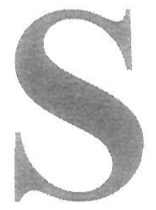

e presentan los primeros resultados de una investigación en avanzado proceso de análisis de los resultados obtenidos. Se analiza una muestra de 98 personas ancianas con problemas de salud y dependencia, que sufren algún tipo de negligencia o de maltrato y que son atendidas por el Servicio de Atención Domiciliaria de los ayuntamientos de Vitoria, Sevilla y los municipios canarios de Las Palmas, Telde y San Bartolomé de Tirajana. Son 2.351 personas ancianas las atendidas en dichos municipios. El total de 111 casos detectados por las auxiliares domiciliaras supone el 4'7\% del total, y eso sin duda es "la punta del iceberg" del problema real.

Entre los resultados destaca que son más comunes los casos de negligencia que los de malos tratos propiamente dichos; que los niveles de los distintos tipos de dependencia/independencia por género son similares aunque las mujeres son algo más dependientes que los varones en cuanto al vestido y control de esfínteres, y algo más independientes en cuanto a movilidad o en la capacidad para alimentarse; que las mujeres son más víctimas de los malos tratos que los varones, incluso aún cuando, como en el caso del maltrato emocional y del abuso material, se trate de mujeres con independencia para vestirse y moverse.

Una conclusión es que la naturaleza de los distintos tipos de malos tratos es diferente, y que parecen afectar a personas con características variadas. Otra es que la dependencia puede conllevar negligencia y/o maltrato. Y quizá la más significativa en cuanto a las políticas de prevención e intervención: que el género -ser mujer- puede ser un factor de riesgo más influyente que sufrir dependencia.

Title: Family violence against the frail and dependent elderly. 
The first results of a research in progress about elder neglect and mistreatment are presented. A sample of 98 abused elderly suffering from some sort of health problems and dependence are examined. They live at home in the Spanish cities of Vitoria and Sevilla, as well as Las Palmas, Telde and San Bartolomé de Tirajana, in the Canary Islands. Home help is provided by the Formal Services to these elderly. 111 mistreatment cases have been detected by the home workers among 2,351 persons assisted by the Community Services in these towns, which amounts to 4.7 per cent. This could be just the tip of the iceberg.

Some results are pointed out: a) neglect appears to be more frequent than mistreatment; b) dependence/independence levels are similar both for men and women, although women are slightly more dependent than men for dressing and sphincters controlling, as well as moderately more independent for moving or self-feeding; c) women are more likely to become victims of mistreatment than men are, especially in the cases of emotional mistreatment and material abuse, among those women who are independent for dressing and moving.

Three main conclusions emerge: a) the nature of mistreatment differs by types; b) neglect and mistreatment may be associated with dependence; and, the most relevant conclusion regarding (with reference to) prevention and intervention policies is that: c) gender, i.e., being a woman, may be a more influential risk factor than being dependent.

\section{INTRODUCCIÓN}

Se presentan algunos resultados de una investigación sobre negligencia y malos tratos a las personas ancianas residentes en la comunidad, realizada por la autora y financiada por el Ministerio de Educación y Cultura (CIYT). Son muchas las dificultades implícitas al propio objeto de la investigación. Quizá es por lo que es la primera vez que se aborda este problema en España. Analizar la violencia familiar ha sido siempre difícil por ser un tema tabú. Durante la década de los años setenta se hace socialmente visible el problema de la violencia entre cónyuges, y en los años ochenta se afianza el término "violencia doméstica", que es más utilizado por los movimientos feministas, y con el que se intenta transmitir la idea de que se produce una violencia dentro del hogar, que es perpetrada por los varones contra las mujeres y los niños. El maltrato a las personas ancianas es un problema complejo y multidimensional que se engloba dentro del más amplio de la violencia familiar, y no existe consenso, según las investigaciones realizadas, acerca de la existencia de unas características personales y/o familiares particulares, que puedan conllevar una probabilidad mayor de con- 
vertirse en víctima o en perpetrador del abuso, ya que los hallazgos de las diferentes investigaciones son diversos y a veces contrarios, aunque se encuentren rasgos comunes (F. Glendeninng, 1993: 14). Tampoco existe una teoría que ofrezca una expíicación comprensiva del problema. Parece no obstante que ciertas teorías sociológicas proporcionan un marco estructural que resulta útil a la Psicología y a la Psicología Social. Son pioneros en el análisis estructural de la violencia familiar los sociólogos estadounidenses Richard J. Gelles y Murray A. Straus (1988). Las teorías feministas, por su parte, han proporcionado un punto de vista fundamental para la comprensión de la violencia familiar, aunque la perpetrada contra las personas ancianas no parece encontrar en ese marco la explicación más completa. No obstante, ha contribuido a complementarla (S. Biggs, Ch. Phillipson y P. Kingston, 1995).

El maltrato a las personas de edad fue detectado desde mediados de los años setenta, pero no es hasta los ochenta en los Estados Unidos cuando se institucionaliza el término de elder abuse. Es el último descubrimiento de la violencia familiar y se entiende que es también el fenómeno violento que todavía en la actualidad sigue siendo ampliamente desconocido (G. Bennett, P. Kingston y B. Penhale, 1997: 1). Obtener información sobre el problema ha resultado una tarea difícil pues, aunque los malos tratos son detectados por los profesionales de la salud y de los servicios sociales, rara vez se prestan a colaborar. Al no existir normativa ni protocolos temen enfrentarse a solas con el problema.

\section{METODOLOGÍA}

A fin de obtener un acercamiento a esa realidad, se solicitó la cumplimentación de un cuestionario por las auxiliares domiciliarias de las empresas que contratan los ayuntamientos de Vitoria, Sevilla y los municipios canarios de Las Palmas, Telde y San Bartolomé de Tirajana. Por defecto de información significativa, se han desechado algunos cuestionarios del total de 111 casos detectados de personas que sufren desatención o maltrato, de entre un grupo de 2.351 personas mayores atendidas por los Servicios de Atención Domiciliaria (SAD) de dichos municipios. Se ha analizado para el presente artículo una muestra de 98 casos de personas de 60 y más años que sufren desatención y/o algún tipo de maltrato. Puede comentarse como primer resultado que el total de 111 casos detectados suponen el 4'7\% del total de las personas atendidas, lo que quizá sólo sea la punta del iceberg de la realidad social.

Dado que en diversas investigaciones suele aparecer el género, la 
discapacidad y el estrés de la persona cuidadora como elementos asociados con el maltratro a las personas de edad, las hipótesis que dirigen el desarrollo de la investigación son: a) la dependencia de la persona anciana conlleva una probabilidad mayor de sufrir negligencia y/o malos tratos; b) las mujeres son más víctimas de malos tratos (también) en su ancianidad que los varones; c) la dependencia económica de la persona cuidadora conduce al maltrato de sus familiares ancianos. Se ha distinguido entre negligencia y maltrato, entendiéndose la primera como la desatención de las necesidades físicas y psicológicas de la persona anciana. El maltrato es una acción intencionada dirigida a infligir un daño a la persona anciana. Puede ser maltrato físico, maltrato psíquico, abuso material y abuso sexual ${ }^{1}$.

\section{RESULTADOS}

Todas las personas que se analizan son víctimas de algún tipo de negligencia o de maltrato, al tiempo que presentan diferentes tipos de dependencia y de enfermedad. La dependencia se ha medido por la dificultad de las personas para ser autónomas en diversas actividades de la vida diaria: dificultades para el vestido, para el aseo personal, en la movilidad, alimentación, y control de esfínteres. Es para el aseo personal donde se observa una mayor dificultad ( $77 \%$ del total), seguido de la movilidad (69\%), vestido (60\%), alimentación (59\%) y control de esfínteres $(35 \%)$.

Por género, se observan similitudes y diferencias en cuanto a los tipos de dependencia. Así, aunque las mujeres de la muestra parecen algo menos autónomas que los varones en cuanto al vestido y el control de esfínteres, y algo más que ellos en cuanto a la movilidad, las diferencias no son notables en ninguna de las actividades de la vida cotidiana, excepto en la capacidad para alimentarse, que es menor entre los varones. Es en este aspecto en el que los varones aparecen más dependientes que las mujeres.

Del total de la muestra analizada, son más las personas que se observa que sufren negligencia que malos tratos. El 70\% sufre negligencia en el cuidado físico y el $47 \%$ en el cuidado psicoafectivo, el $8 \%$ maltrato físico, el $29 \%$ maltrato psíquico o emocional, y el $18 \%$ abuso material. La mayor proporción de personas en las que aparecen signos de desatención es en los aspectos físicos. Los aspectos afectivos deteriorados que muestran las carencias de un trato adecuado ocurren de forma mayoritaria, y no tanto el maltrato físico en sí, aunque en varias

Entre los 111 casos apareció uno de abuso sexual de una mujer con graves problemas de dependencia física, por parte de su marido, mayor que ella. 
personas se dan diferentes tipos de negligencia y/o maltrato al mismo tiempo. Aunque en todas las edades el respeto, el cariño y la atención prestada son aspectos fundamentales para el desarrollo armónico de cualquier ser humano, tratándose de personas mayores, enfermas, y/o con problemas de dependencia, los aspectos afectivos tienen una importancia singular en su calidad de vida.

Por género, como se aprecia en la tabla 1, aparecen unas diferencias interesantes. Dado que el tipo de abuso observado en mayor medida en todas las personas de la muestra es la negligencia en el cuidado físico, es lógico que las proporciones mayores por género sean en este tipo de abuso, aunque los varones aparecen sufriendo más descuido o desatención física que las mujeres, y es que como se ha señalado, en cuanto a movilidad, ellos son algo más dependientes (74\%) que las mujeres $(68 \%)$, pero sobre todo en cuanto a la capacidad para alimentarse (74\% frente al $55 \%$ de las mujeres), por lo que pueden aparecer más desnutridos y en peor estado físico. En cuanto a la negligencia en el cuidado psicoafectivo, las proporciones son similares, sin embargo, las diferencias entre géneros son desproporcionadas en cuanto al maltrato en sí. En el de tipo físico -aunque la proporción general es pequeña, pero precisamente por eso mismo resulta reseñable- las mujeres lo sufren en una proporción del doble de los varones. En el de tipo psicológico o emocional, algo más de uno de cada diez varones lo sufre, mientras entre las mujeres son víctimas del mismo tres de cada diez. $Y$ en el abuso material la proporción de mujeres que lo padecen es seis veces la de los varones.

Tabla 1: Tipos de negligencia o abuso por género (en porcentajes)

\begin{tabular}{|l|c|c|}
\hline Tipo de negligencia o maltrato & Varones & Mujeres \\
\hline Negligencia en el cuidado físico & 78 & 68 \\
\hline Negligencia en el cuidado psicoafectivo & 44 & 48 \\
\hline Maltrato físico & 4 & 9 \\
\hline Maltrato psicológico o emocional & 13 & 33 \\
\hline Abuso material & 4 & 23 \\
\hline Total (\%) & 100 & 100 \\
\hline Total (N) & 23 & 75 \\
\hline
\end{tabular}

Analizando en la tabla 2 la posible asociación entre tipo de dependencia en las actividades de la vida cotidiana y tipo de negligencia o maltrato sufridos, se puede decir que, al comparar los tipos de dependencia con la experiencia de negligencia en el cuidado físico, parecen 
variables influyentes la dependencia para vestirse, asearse y parece que especialmente para alimentarse. No se aprecia diferencia notable en cuanto a la negligencia en el cuidado físico entre quienes tienen problemas de movilidad y quienes no los tienen, ni sobre todo entre quienes controlan los esfínteres y quienes no los controlan, ya que todas esas personas sufren en proporciones parecidas, y también altas, la falta de atención en el cuidado físico. Parece pues que en ambos tipos de actividad coinciden otras características que neutralizan el "efecto beneficioso" de la independencia como preventivo de sufrir negligencia en el cuidado físico.

La negligencia en el cuidado psicoafectivo es el segundo tipo de abuso que sufren más las personas de la muestra. Entre quienes tienen dificultades en alguna de las actividades de la vida cotidiana, parece que aquellas personas que son dependientes de otras para el aseo personal, la alimentación y el control de esfínteres son las que pueden sufrir más este tipo de negligencia. Sobre todo en este último tipo de dependencia (dificultad para el control de esfínteres) donde la diferencia porcentual en la experiencia de este tipo de abuso es la mayor, y la única que supera el 50\%. Parece posible que la necesidad de limpieza corporal, la mayoría de las veces por parte de otras personas, pueda conllevar por parte de quien la realiza actitudes y gestos de disgusto, desagrado y reproche hacia la persona dependiente. En las otras dos actividades (vestido y movilidad) no se observan diferencias en cuanto a ser dependiente o independiente. Es decir, se sufre en proporciones iguales o similares la negligencia de carácter psicoafectivo. Se puede recordar que por género las proporciones son similares en cuanto a padecer carencia de atención afectiva.

Respecto al maltrato físico, hay que tener en cuenta que sólo en 8 casos se ha observado, pero algunos aspectos llaman la atención. Precisamente entre las personas que son autónomas para el vestido lo sufre un $12 \%$ de las mismas y un $5 \%$ de quienes son dependientes, así como el $10 \%$ de quienes controlan los esfinteres frente al 3\% de quienes son dependientes. De esta primera visión, parecería que al menos en esas dos actividades la autonomía no prevendría del maltrato físico. Puede que otras variables influyan en esa situación, aunque el escaso número de personas en las que se ha observado maltrato físico lleva a la prudencia a la hora de elaborar hipótesis explicativas. En cuanto a la capacidad para el aseo personal no aparece nadie maltratado entre quienes son autónomos en esa actividad. En el resto de actividades no se observan diferencias. En el maltrato psicoafectivo llama también la atención que el $46 \%$ de quienes no tienen problemas para vestirse sufra ese tipo de maltrato, frente al $19 \%$ de quienes tienen problemas. Algo parecido aunque en menor proporción ocurre con quienes no tie- 
nen problemas para alimentarse que sufren maltrato psicoafectivo en una proporción del $38 \%$ frente al $22 \%$ de quienes dependen de alguien. En el resto de las actividades no se observan diferencias. En relación al abuso material, el $21 \%$ de las personas que son autónomas para vestirse sufre ese tipo de abuso, frente al $12 \%$ de quienes son dependientes. Algo parecido ocurre a quienes no tienen problemas de movilidad, pues el $21 \%$ del grupo sufre este tipo de abuso frente al $13 \%$. A primera vista parece algo contradictorio que quienes son más autónomas para vestirse y moverse - lo que implicaría poder salir de casa al Banco, a la notaría o a pedir cualquier tipo de asesoramiento, así como ocuparse de los propios asuntos económicos y materiales en generalsean precisamente quienes sufran más esos tipos de abuso con respecto a las dependientes. Otros factores influyen sin duda en que esas personas sean sometidas a tales tipos de abuso.

Resumiendo, en la negligencia en el cuidado físico parece que en general influye la dependencia de la persona anciana para las actividades de la vida cotidiana. En la negligencia en el cuidado psicoafectivo parece influir la falta de control de los esfínteres y también las dificultades para el aseo personal. En el maltrato emocional no parece haber relación entre sufrir este tipo de maltrato y padecer dependencia, ya que o bien las personas que son autónomas lo sufren en la misma proporción que las dependientes, o bien incluso en una proporción mayor todavía que ellas. Lo mismo ocurre con el abuso material aunque las diferencias porcentuales entre personas autónomas y personas dependientes sean menores, pero justamente son las personas que teóricamente tienen más posibilidades de salir a la calle las que sufren más abuso.

Si se vuelve a analizar la tabla $l$ se observa que es en los tipos de maltrato psicológico o emocional y abuso material donde las diferencias entre varones y mujeres es notable. Son las mujeres las que sufren de manera desproporcionada esos tipos de malos tratos, dejando de lado el maltrato físico por el pequeño número de casos observados (ocho) pero de los que en siete de ellos las víctimas son mujeres. Al analizar la violencia familiar debe tenerse en cuenta además del edadismo existente el componente sexista (L. Aitken y G. Griffin, 1996).

Profundizando más en el análisis entre enfermedad y malos tratos se estudia la asociación entre abusos y "demencia" y "otra enfermedad incapacitante". Se deja de lado por la escasa frecuencia de casos, la posibilidad de sufrir otra enfermedad mental y hemiplejia. En cuanto a la negligencia en el cuidado físico no se observa ninguna asociación con los citados tipos de enfermedad. Sin embargo, en la negligencia en el cuidado psicoafectivo, la proporción de quienes tienen demencia y 
sufren ese tipo de desatención (74\%) es notablemente mayor que la de quienes no sufren demencia (42\%). Parece que los comportamientos relacionados con la demencia podrían influir en que la persona cuidadora desatendiera las necesidades afectivas de la persona enferma, por causa del propio estrés y otros problemas de convivencia familiar que suelen producirse (Bazo, 1998). La asociación resulta inversa en cuanto a sufrir otra enfermedad incapacitante: el $63 \%$ de quienes no la padecen es víctima de ese tipo de negligencia, frente al $44 \%$ de quienes sí la padecen. Por otro lado, sufrir demencia puede influir en sufrir maltrato emocional (37\%) pues la proporción de maltratados entre quienes no sufren demencia es menor (24\%). Ahora bien, las proporciones se invierten entre quienes no sufren otra enfermedad invalidante, pues entre ellas sufre maltrato emocional una proporción del $38 \%$ frente al $22 \%$ de quienes sufren otros tipos de incapacitación. De nuevo se observan en las relaciones entre enfermedad y maltrato ciertas contradicciones.

Respecto al abuso material las proporciones son relativamente similares tanto si se sufre demencia $(21 \%)$ como si no (18\%) y son las personas que no sufren otra enfermedad invalidante $(25 \%)$ las que proporcionalmente sufren más abuso material que las otras $(16 \%)$. Comparando estos tipos de enfermedad en relación a la negligencia y maltrato sufridos, las frecuencias son notablemente más bajas que al asociar los malos tratos con los tipos de dependencia para las actividades de la vida diaria. Puede deberse a que las observaciones han sido realizadas por personal no experto en salud, sino por auxiliares domiciliarias. Esas personas pueden juzgar con más acierto las discapacidades de las personas en relación a las actividades de la vida diaria que, obviamente, ciertos tipos de enfermedad. Resumiendo, parece que la demencia y la carga que conlleva para la persona cuidadora puede influir en sufrir negligencia psicoafectiva y maltrato emocional.

Tabla 2. Tipos de negligencia y maltrato por tipos de dependencia

\begin{tabular}{|l|r|r|r|r|r|r|r|r|r|r|}
\hline & \multicolumn{2}{|c|}{ Vestido } & \multicolumn{2}{c|}{ Aseo Personal } & \multicolumn{2}{c|}{ Movilidad } & \multicolumn{2}{|c|}{ Alimentación } & \multicolumn{2}{c|}{ Control Esfint. } \\
\cline { 2 - 14 } & Sí & No & Sí & No & Sí & No & S í & No & S í & No \\
\hline Negligencia cuidado físico & 85 & 49 & 76 & 53 & 74 & 67 & 79 & 59 & 74 & 71 \\
\hline Negligenciacuidadopsicoafectivo & 44 & 46 & 47 & 35 & 46 & 42 & 48 & 38 & 56 & 38 \\
\hline Maltrato físico & 5 & 12 & 9 & -- & 9 & 4 & 9 & 6 & 3 & 10 \\
\hline Maltrato psicoafectivo & 19 & 46 & 29 & 24 & 27 & 33 & 22 & 38 & 27 & 29 \\
\hline Abuso material & 12 & 21 & 15 & 18 & 13 & 21 & 19 & 9 & 12 & 17 \\
\hline
\end{tabular}


Tratando de profundizar más en el tema y de asegurar la importancia de otros factores, como es el género, que puedan ayudar a explicar más esa especie de contradicciones (sufrir más negligencia o maltrato quienes son autónomas en ciertas actividades que quienes son dependientes), dado que las mujeres son más víctima de malos tratos, se realizan otros tipos de análisis. Se ha realizado una neutralización de variables, tratando de encontrar la relación entre el abuso material y el maltrato psicológico o emocional con el género, controlando la influencia de la dependencia para vestirse y moverse.

De los datos obtenidos se aprecia el "factor de riesgo" que supone ser mujer. Puede decirse que entre quienes tienen dificultades para vestirse, el $7 \%$ de los varones sufre abuso material, así como el $13 \%$ de las mujeres. Entre quienes no tienen dificultades, ningún varón sufre abuso material, pero sí lo sufre el $30 \%$ de las mujeres. Entre quienes tienen dificultades de movilidad, ningún varón sufre abuso material, pero sí el 18\% de las mujeres. Entre quienes son autónomos en esa actividad, el $17 \%$ de los varones sufre abuso material y también el $22 \%$ de las mujeres. Entre quienes tienen dificultades para vestirse ningún varón sufre maltrato psicológico, mientras lo sufre el $24 \%$ de las mujeres. Entre quienes son independientes para esta actividad el $30 \%$ de los varones sufre ese tipo de maltrato, pero más de la mitad de las mujeres $(52 \%)$ también son víctimas del mismo. Entre quienes tienen problemas de movilidad el $18 \%$ de los varones sufre maltrato psicológico o emocional, así como el $29 \%$ de las mujeres. Entre quienes no experimentan ese tipo de dependencia ningún varón sufre maltrato psicológico, aunque sí lo sufre casi la mitad de las mujeres (44\%).

\section{CONCLUSIONES}

En la presente investigación se encuentra que, siendo todas las personas de la muestra víctimas de alguno o varios tipos de maltrato, al tiempo que dependientes en diferente grado de otras personas para la realización de las actividades de la vida diaria, la naturaleza de los tipos de malos tratos es diferente, y afectan a personas con características distintas. Es decir, que sufrir dependencia en algunas actividades de la vida cotidiana puede conllevar descuido físico o psicológico, sin embargo, ser autónoma en actividades como el vestido, alimentación o movilidad puede conllevar maltrato psicoafectivo y abuso material en mayor proporción que entre quienes son dependientes. El género aparece en esos dos últimos tipos más importante que la dependencia. La negligencia en el cuidado físico que implica una falta de atención física, material, ocurre más entre quienes tienen dificultades o dependen de 
otras personas para vestirse, asearse, y alimentarse. La negligencia en el cuidado psicoafectivo que conlleva falta de cariño y detalles afectuosos parece darse más entre quienes son dependientes para su aseo personal, alimentarse y controlar los esfínteres. Ambos tipos de negligencia se relacionan. Pueden ser más descuidadas y desatendidas física y afectivamente las personas que dependen de otras para su cuidado personal, lo que implica el cuidado corporal. No puede olvidarse el significado simbólico del cuerpo ni su atractivo o repulsión en función de la edad o la enfermedad. Son de naturaleza diferente el maltrato psicoafectivo y el abuso material, más relacionados con el hecho de ser mujer que con ciertos tipos de dependencia física.

Las mujeres proporcionalmente sufren más malos tratos físicos y psicológicos, y abuso material que los varones, y negligencia de forma parecida, aunque los tipos de dependencia que sufren son parecidos a los de los varones, excepto en la capacidad para alimentarse que son notablemente más autónomas que ellos. Parece pues que influye más en el hecho de sufrir maltrato el ser mujer que el ser dependiente. En cuanto a la posible asociación entre sufrir ciertas enfermedades y ser víctima de malos tratos, parece que la demencia puede conllevar negligencia psicoafectiva y maltrato emocional. Además, a pesar de que se han observado en menor frecuencia esos casos que los relacionados con la dependencia, sin embargo, también aquí se observan asociaciones inversas entre tipos de enfermedad (otra enfermedad incapacitante) y malos tratos.

Al profundizar en el análisis relacionando sufrir maltrato psicoafectivo así como abuso material con el género, pero neutralizando la influencia de la capacidad/incapacidad para vestirse, así como moverse, se aprecia que ser mujer se asocia con el sufrimiento de esos tipos de maltrato. Su menor poder social y aislamiento de la esfera pública las hace a toda edad más vulnerables. De la neutralización de variables puede concluirse que la dependencia para vestirse puede influir en sufrir abuso material, pero es más importante la circunstancia de ser mujer. También es más importante ser mujer a la hora de sufrir maltrato psicológico que tener dificultades para vestirse. Por otro lado, sufrir abuso material entre quienes tienen dificultades para moverse sólo parece asociarse con ser mujer, que también pueden sufrirlo aún siendo independientes para esa actividad. Ocurre lo mismo en cuanto a sufrir maltrato psicológico o emocional. Parece que influye más ser mujer que los problemas de movilidad. Ser mujer es un factor (cultural) de riesgo de sufrir malos tratos a lo largo de toda la vida, pues pueden sufrirlos desde la cuna a la tumba. Finalmente, la hipótesis de que experimentar dependencia puede conllevar sufrir más algún tipo de negligencia y/o maltrato parece cierto, al menos, en algunos tipos, pero pa- 
rece resultar más sólida la hipótesis de que en ciertos casos ser mujer es un factor de riesgo más alto que ser físicamente dependiente.

\section{DISCUSIÓN}

Soy consciente de la limitación relativa de los datos manejados, aunque queda por analizar en la investigación algún otro aspecto como las características de las personas supuestamente maltratadoras. Sin embargo, podría ser mucho más clarificador poder realizar un estudio cualitativo entre las personas maltratadas y sus maltratadores. Las dificultades en la actualidad en España para realizar ese propósito son enormes dado que no existe una conciencia social, ni por tanto política, sobre el problema. Eso conlleva la falta de directrices para los propios servicios sociales y sanitarios que no les queda más actuación que la vía judicial ante los casos más extremos. Pero es un objetivo que estoy determinada en lograr.

Por otro lado, es cierto que la muestra analizada es precisamente de personas en las que se ha observado que son víctimas de algún tipo o tipos de negligencia y/o malos tratos, que lo ideal sería poder comparar dos muestras representativas de dos poblaciones que, con características similares de edad, género, situación familiar y niveles de dependencia y enfermedad, unas personas sufran maltrato y otras no. Buscando ese ideal seguiré adelante, consciente de las dificultades que conlleva en nuestra sociedad una investigación de las características de la presente.

Que sean los primeros datos existentes en España sobre maltrato familiar a las personas ancianas es sólo un primer paso para seguir investigando, tratando de comprobar en muestras más amplias y diferentes las hipótesis que aquí parecen confirmarse, y otras, todo lo cual pueda permitir elaborar políticas de prevención de los malos tratos a las personas ancianas, así como de intervención, no sólo en el entorno familiar, sino también en el institucional.

\section{AGRADECIMIENTOS}

Deseo expresar mi agradecimiento a los ayuntamientos, empresas de servicios y auxiliares que me han prestado su apoyo, sin cuya colaboración no hubiera podido realizarse la investigación. 


\section{BIBLIOGRAFÍA}

AITKEN, L. y GRIFFIN G.: 1996. Gender Issues in Elder Abuse, Londres, SAGE. BAZO, M.T.: 1998. "Vejez dependiente, políticas y calidad de vida", Papers. Revista de Sociología, 56: 143-161.

BENNETT, G.; KINGSTON, P.; PENHALE, B.: 1997. The Dimensions of Elder Abuse: Perspectives for Practitioners, Londres, Macmillan.

BIGGS, S.; PHILLIPSON, C.; KINGSTON, P.: 1995. Elder Abuse in Perspective. Buckingham, Open University Press.

GLENDENNING, F.: 1993. "What is elder abuse and neglect?" en Peter Decalmert y Frank Glendenning (eds.) The Mistreatment of Elderly People, Londres, SAGE: 1-34.

GELLES, R.J. y MURRAY A.S.: 1988. Intimate Violence, Nueva Yourk, Simon and Schuster. 